\section{OPEN ACCESS}

Edited by:

Philippe Saas,

INSERM U1098 Interactions

Hôte-Greffon-Tumeur \& Ingénierie

Cellulaire et Génique, France

Reviewed by:

Paolo Puccetti,

University of Perugia, Italy

Julio Villena,

Centro de Referencia para

Lactobacilos, Argentina

Siegfried Hapfelmeier,

Universität Bern, Switzerland

*Correspondence:

Alain P. Gobert

alain.p.gobert@vanderbilt.edu;

Keith T. Wilson

keith.wilson@vanderbilt.edu

tThese authors have contributed equally to this work.

Specialty section:

This article was submitted to Inflammation,

a section of the journal

Frontiers in Immunology

Received: 15 March 2018

Accepted: 17 May 2018

Published: 05 June 2018

Citation:

Gobert AP, Al-Greene NT, Singh K, Coburn LA, Sierra JC, Verriere TG,

Luis PB, Schneider C, Asim M, Allaman MM, Barry DP, Cleveland JL, Destefano Shields CE, Casero RA Jr.,

Washington MK, Piazuelo MB and Wilson KT (2018) Distinct

Immunomodulatory Effects of Spermine Oxidase in Colitis Induced by Epithelial Injury or Infection.

Front. Immunol. 9:1242. doi: 10.3389/fimmu.2018.01242

\title{
Distinct Immunomodulatory Effects of Spermine Oxidase in Colitis Induced by Epithelial Injury or Infection
}

\begin{abstract}
Alain P. Gobert', ${ }^{1,2}$, Nicole T. Al-Greene ${ }^{1 \dagger}$, Kshipra Singh ${ }^{1,2}$, Lori A. Coburn ${ }^{1,2,3}$, Johanna C. Sierra ${ }^{1,2}$, Thomas G. Verriere ${ }^{1}$, Paula B. Luis', Claus Schneider ${ }^{2,4}$, Mohammad Asim', Margaret M. Allaman', Daniel P. Barry', John L. Cleveland ${ }^{5,6}$, Christina E. Destefano Shields ${ }^{7}$, Robert A. Casero Jr. ${ }^{7}$, M. Kay Washington ${ }^{8}$, M. Blanca Piazuelo ${ }^{1,2}$ and Keith T. Wilson ${ }^{1,2,3,8 *}$
\end{abstract}

\begin{abstract}
Division of Gastroenterology, Hepatology, and Nutrition, Department of Medicine, Vanderbilt University Medical Center, Nashville, TN, United States, ${ }^{2}$ Center for Mucosal Inflammation and Cancer, Vanderbilt University Medical Center, Nashville, TN, United States, ${ }^{3}$ Veterans Affairs Tennessee Valley Healthcare System, Nashville, TN, United States, ${ }^{4}$ Department of Pharmacology, Vanderbilt University Medical Center, Nashville, TN, United States, ${ }^{5}$ Department of Tumor Biology, Moffitt Cancer Center and Research Institute, Tampa, FL, United States, ${ }^{6}$ Department of Cancer Biology, The Scripps Research

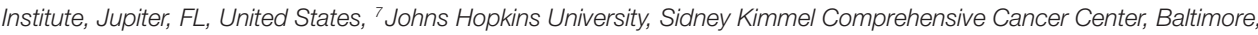
MD, United States, ${ }^{8}$ Department of Pathology, Microbiology, and Immunology, Vanderbilt University Medical Center, Nashville, TN, United States
\end{abstract}

Polyamines have been implicated in numerous biological processes, including inflammation and carcinogenesis. Homeostatic regulation leads to interconversion of the polyamines putrescine and the downstream metabolites spermidine and spermine. The enzyme spermine oxidase (SMOX), which back-converts spermine to spermidine, contributes to regulation of polyamine levels, but can also have other effects. We have implicated SMOX in gastric inflammation and carcinogenesis due to infection by the pathogen Helicobacter pylori. In addition, we reported that SMOX can be upregulated in humans with inflammatory bowel disease. Herein, we utilized Smox-deficient mice to examine the role of SMOX in two murine colitis models, Citrobacter rodentium infection and dextran sulfate sodium (DSS)-induced epithelial injury. In C. rodentium-infected wild-type (WT) mice, there were marked increases in colon weight/length and histologic injury, with mucosal hyperplasia and inflammatory cell infiltration; these changes were ameliorated in Smox ${ }^{-1-}$ mice. In contrast, with DSS, Smox ${ }^{-1-}$ mice exhibited substantial mortality, and increased body weight loss, colon weight/length, and histologic damage. In C. rodentium-infected WT mice, there were increased colonic levels of the chemokines CCL2, CCL3, CCL4, CXCL1, CXCL2, and CXCL10, and the cytokines IL-6, TNF- $\alpha$, CSF3, IFN- $\gamma$, and IL-17; each were downregulated in Smox ${ }^{-1-}$ mice. In DSS colitis, increased levels of IL-6, CSF3, and IL-17 were further increased in Smox ${ }^{-1-}$ mice. In both models, putrescine and spermidine were increased in WT mice; in Smox ${ }^{-1-}$ mice, the main effect was decreased spermidine and spermidine/spermine ratio. With C. rodentium, polyamine levels correlated with histologic injury, while with DSS, spermidine was inversely correlated with injury. Our studies indicate that SMOX has immunomodulatory effects in experimental colitis via polyamine flux. Thus, SMOX contributes to the immunopathogenesis of $C$. rodentium infection, but is protective in DSS colitis, indicating the divergent effects of spermidine.

Keywords: colitis, infection, polyamines, spermidine, spermine oxidase, mucosal immune response 


\section{INTRODUCTION}

Polyamines are pleiotropic and abundant aliphatic molecules found in all mammalian cells. Putrescine, spermidine, and spermine bind both RNA and DNA and are involved in various cellular processes such as maintenance of DNA structure, gene expression, RNA folding and bending, and protein translation (1-3). These three biogenic polyamines are thus essential for cell homeostasis, growth, differentiation, division, and death $(4,5)$. In addition, polyamines exhibit critical effects during pathological conditions through their potent toxicity at high concentration (6), regulation of inflammation $(7,8)$, and modulation of oxidative stress $(9,10)$. In this context, the homeostasis of polyamine content within a non-toxic range through synthesis, degradation, and transport is a considerable challenge for cells.

Putrescine, the first polyamine in the anabolic pathway, is generated by ornithine decarboxylation catalyzed by cytosolic ornithine decarboxylase 1 (ODC1, also known as ODC). Putrescine is then sequentially converted into spermidine and spermine via the action of spermidine synthase and spermine synthase, respectively (5). Importantly, the back conversion of spermine to spermidine and putrescine is a critical step in the regulation of polyamine levels, notably to limit the cytotoxic effects of spermine accumulation (11). This can be achieved by two metabolic pathways: first, the enzyme spermidine/ spermine $N(1)$-acetyltransferase 1 catalyzes the acetylation of spermine into $N(1)$-acetylspermine and spermidine into $N(1)$-acetylspermidine; and then, the oxidation of these two products through the enzyme polyamine oxidase leads to the formation of spermidine and putrescine, respectively. However, this pathway is poorly expressed in the gastrointestinal tract during infection and inflammation (12-14). Second, the flavoenzyme spermine oxidase (SMOX) directly catalyzes the oxidation of spermine to spermidine and yields the generation of 3-aminopropanal and $\mathrm{H}_{2} \mathrm{O}_{2}$ (15). This enzyme is expressed in macrophages $(9,14,16)$ and epithelial cells $(9,16,17)$ during infection of the gastrointestinal tract. Moreover, it has been described that the generation of $\mathrm{H}_{2} \mathrm{O}_{2}$ by SMOX regulates DNA damage and apoptosis, thus supporting the development of gastric carcinoma during Helicobacter pylori infection (17-19) and Bacteroides fragilis-induced colon tumorigenesis in Min mice (16). It is important to underline that these data have been obtained by using the pharmacological SMOX inhibitor MDL 72527.

Inflammatory bowel disease (IBD) afflicts nearly two million people in the USA and is increasing worldwide $(20,21)$. Despite implementation of various biologic therapies, less than half of patients achieve remission. Increased understanding of the potential role of polyamines and SMOX is warranted, given the prior studies implicating SMOX in gastric inflammation and cancer $(18,19)$ and in human IBD $(22)$. In this work, we questioned whether SMOX has an important role in the development of colitis. To answer this directly, we used Smox ${ }^{-/-}$mice and two models of experimental colitis: infection with Citrobacter rodentium and treatment with dextran sulfate sodium (DSS).

\section{MATERIALS AND METHODS}

\section{Animals and Models of Colitis}

Experiments were conducted under protocol M/08/124 approved by the Vanderbilt University IACUC and Institutional Biosafety Committee, and the Research and Development Committee of the Veterans Affairs Tennessee Valley Healthcare System. Procedures were performed in accordance with institutional policies, AAALAC guidelines, the AVMA Guidelines on Euthanasia ( $\mathrm{CO}_{2}$ asphyxiation followed by cervical dislocation), NIH regulations (Guide for the Care and Use of Laboratory Animals), and the United States Animal Welfare Act (1966).

C57BL/6 and C57BL/6 Smox ${ }^{-/-}$mice (23) were house-bred in our animal facility. Age-matched male wild-type (WT) and mutant mice (8-12 weeks) were used for two models of colitis: (1) mice were infected by oral gavage with $0.1 \mathrm{ml}$ of LB broth containing $5 \times 10^{8}$ C. rodentium DBS100 (24) as reported (25-27); feces were collected during the time course of infection and animals were euthanized after 14 days. (2) Animals were treated with 2.5\% DSS (mol. wt. 36,000-50,000; TdB Consultancy) in the drinking water for 5 days; DSS was then removed, and mice were kept for 5 more days before euthanasia. In both models, mice were weighed and monitored daily and those that showed extreme distress, became moribund, or lost more than $20 \%$ of initial body weight were euthanized. After sacrifice, colons were removed, measured, cut longitudinally, cleaned, weighed, and Swiss-rolled for histology. Three proximal and distal 2-mm pieces were used for RNA and protein analysis and to determine C. rodentium colonization by culturing serial dilution of ground tissues on Luria-Bertani agar plates (26).

\section{Histological Injury Scores}

Swiss-rolled colons were fixed in formalin and embedded in paraffin, and $5-\mu \mathrm{m}$ sections were stained with hematoxylin and eosin (H\&E) and examined in a blinded manner by gastrointestinal pathologists (M. Blanca Piazuelo and M. Kay Washington). For DSS colitis, inflammation severity (0-3) and inflammation extent $(0-3)$ were each multiplied by the percent involvement $(1=0-25 \%, 2=25-50 \%, 3=50-75 \%$, and $4=75-100 \%)$ and added together to yield the inflammation score $(0-24)$; the parameter of crypt damage (0-4) was also multiplied by the percent involvement to yield an epithelial injury score (0-16). These scores were then added together to yield the histological injury score $(0-40)$, as described $(25,28,29)$. For $C$. rodentium colitis, the histologic injury score (0-21) was the sum of acute and chronic inflammation ( $0-3$ for each) scores multiplied by extent of inflammation (0-3) plus the epithelial injury score (0-3), as described $(25,26)$.

\section{Cytokine and Chemokine Analysis}

Snap-frozen colon tissues were homogenized in CelLytic MT Cell Lysis Reagent (Sigma). Total protein concentration was determined by BCA Protein Assay (Pierce). The lysates were then analyzed using the Milliplex MAP Kit (Millipore) on a Luminex FlexMAP 3D instrument as described (Millipore) $(26,27,30)$. 


\section{Measurement of Polyamines}

The concentration of the three biogenic polyamines was determined by mass spectrometry as reported (27).

\section{Statistics}

Figures and statistics were performed using Prism 7.0a software (Graphpad Inc.). All the data represent the mean \pm SEM. Data that were not normally distributed were log transformed and distribution was assessed. Student's $t$-test or ANOVA with the Tukey test were used to determine significant differences between two groups or to analyze significant differences among multiple test groups, respectively.

\section{RESULTS}

\section{Attenuation of C. rodentium-Induced Colitis with Smox Deletion}

We previously reported that the SMOX inhibitor MDL 72527 reduces $H$. pylori-induced gastritis and carcinogenesis (19). To now determine the role of SMOX in another infectious model of the gastrointestinal tract, we infected WT and $S m o x^{-/-}$mice with $C$. rodentium, the rodent equivalent of enteropathogenic Escherichia coli that causes colitis in mice $(7,26)$. C. rodentium colonization of the colon and levels in the feces were not affected by the deletion of Smox (Figure 1A; Figure S1 in Supplementary Material). When infected with $C$. rodentium, WT mice did not increase their weight to the same degree as uninfected animals (Figure 1B); by contrast, infected $S m o x^{-/-}$mice gained weight as uninfected mice, and there was a significant difference between the body weight of infected WT and infected Smox-deficient mice at day 8 and 10 post-infection (Figure 1B). Moreover, colon weight-to-length ratio, an indicator of inflammation that was increased in infected animals, was significantly attenuated by $59.3 \pm 3.8 \%$ in infected $S m o x^{-/-}$mice compared with infected WT mice (Figure 1C). H\&E staining of the colons of C. rodentium-infected WT mice demonstrated an effacement of the brush border, hyperplasia, severe mucosal inflammation, and submucosal edema (Figure 1D). However, the histologic damage was markedly attenuated in infected $S$ mox ${ }^{-/-}$mice (Figure 1D). Using a comprehensive scoring system to quantify the degree of inflammation and epithelial damage $(25,26)$, we found a significant reduction in histologic injury in $\mathrm{Smox}^{-/-}$mice compared with WT animals (Figure 1E). There was no detectable inflammation or epithelial injury in uninfected $S m o x^{-/-}$or WT mice (Figure 1D).

\section{Smox Deletion Exacerbates Colitis in the DSS Model}

We next treated WT and Smox ${ }^{-/-}$mice with DSS to assess the role of SMOX in a second model of experimental colitis. We used a model of injury and recovery, caused by this epithelial-damaging agent $(28,29)$. Surprisingly, we observed profound mortality beginning on day 6 of the 10-day protocol in the Smox ${ }^{-/-}$mice, which was not observed in DSS-treated WT animals (Figure 2A). Contrary to the $C$. rodentium model, the weight-to-length ratio of the colon was increased in Smox ${ }^{-/-}$mice compared with WT mice treated with DSS (Figure 2B). WT and Smox ${ }^{-/-}$mice began losing weight on day 6 and day 4 after starting DSS, respectively (Figure 2C). However, in accordance with the increased mortality, Smox ${ }^{-/-}$mice lost more body weight than WT mice (Figure 2C). Moreover, we observed increased overall histological injury in DSS-treated Smox ${ }^{-/-}$mice compared with WT mice with DSS colitis, with increased colonic inflammation, epithelial damage/ crypt loss, and crypt abscesses (Figures 2D,E).

\section{The Deletion of Smox Affects the Mucosal Immune Response During Colitis}

To further assess the role of SMOX on the immune response during colitis, we analyzed the synthesis of mediators of the inflammatory response in the colonic mucosa. As shown in Table S1 in Supplementary Material and Figure 3A, the infection of WT mice with $C$. rodentium led to an increased production of the innate cytokines IL-6, TNF- $\alpha$, and CSF3 (also known as G-CSF), as well as the prototype Th1 cytokine IFN- $\gamma$, and the Th17 cytokine IL-17. Each of these cytokines was less abundant in the colons of C. rodentium-infected Smox ${ }^{-/-}$mice compared with infected WT animals (Figure 3A). Similarly, the chemokines CCL2, CCL3, CCL4, CXCL1, CXCL2, and CXCL10 were significantly increased in the colon of $C$. rodentium-infected WT mice compared with uninfected animals (Table S1 in Supplementary Material); these chemokines were not significantly increased in the mucosa of Smox-deficient mice during the infection and were decreased from the levels in the WT mice (Table S1 in Supplementary Material).

IL-6, CSF3, TNF- $\alpha$, and IL-17 were also induced in the colon of DSS-treated WT mice compared to untreated animals (Figure 3B); the levels of induction were comparable to those observed in C. rodentium colitis (Figure 3A). However, IFN- $\gamma$ was not increased in the colonic mucosa in DSS-induced colitis (Figure 3B). Notably, the effectors induced with DSS treatment, except TNF- $\alpha$, were further increased in Smox-deficient mice that were treated with DSS (Figure 3B). The level of IFN- $\gamma$ in the colon was not modulated in animals with Smox deletion (Figure 3B). Only the chemokines CCL2 and CXCL1 were significantly more abundant in the colon of DSS-treated animals, and there was no difference between WT and Smox ${ }^{-/-}$mice (Table S2 in Supplementary Material).

\section{Spermidine and Spermine Concentrations Are Regulated by SMOX in Colitis}

To further understand the role of SMOX, we analyzed the effect of Smox deletion on polyamine concentrations in the colon during C. rodentium and DSS-induced colitis. First, we found that Smox mRNA expression was significantly induced by $2.25 \pm 0.35$-fold in the colon of WT animals treated with DSS compared with untreated mice (Figure 4A); however, Smox upregulation was not observed during $C$. rodentium infection (Figure $4 \mathrm{~A}$ ). The deletion of Smox was associated with a complete elimination of Smox gene expression in the mouse colons in all of the naive and colitis conditions (Figure 4A). The colonic levels of putrescine were higher in both WT and Smox-deficient mice infected with C. rodentium or treated with DSS compared with control animals, 


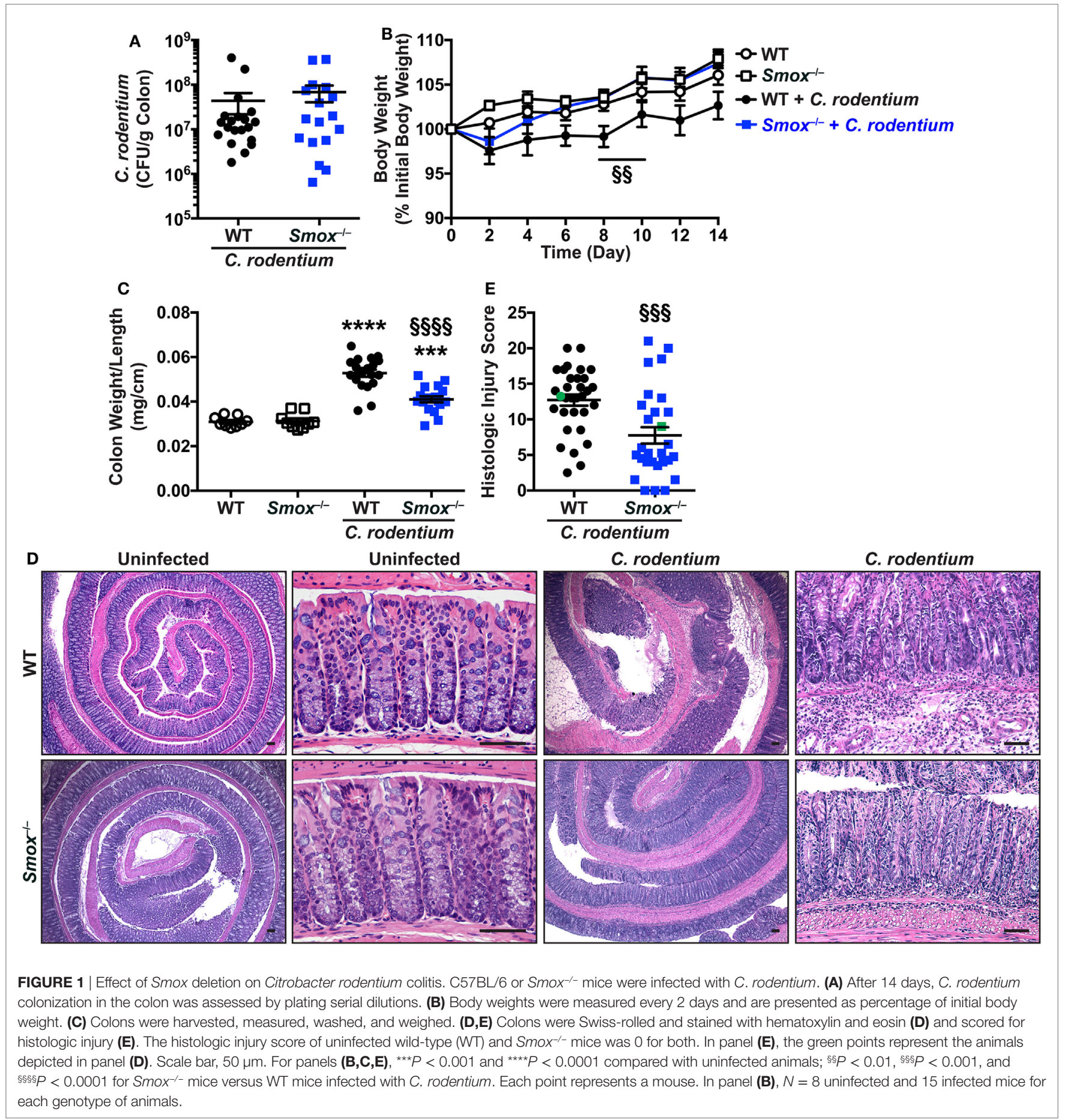

and there was no significant difference between WT and Smox ${ }^{-/-}$ mice (Figure 4B). Spermidine concentration was also increased in both C. rodentium and DSS colitis tissues (Figure 4C). Notably, spermidine levels were significantly reduced by 31 and $54 \%$ in Smox ${ }^{-/-}$mice during C. rodentium infection and DSS treatment, respectively (Figure $4 \mathrm{C}$ ). We did not observe an increase of spermine concentration in the colon of mice with either form of colitis, but there was more spermine in Smox-deficient mice than in WT animals in the C. rodentium model (Figure 4D). There was a modest increase in spermine in Smox-deficient mice treated with DSS, which did not reach statistical significance. Finally, the spermidine/spermine ratio was significantly increased in C. rodentium-infected or DSS-treated WT mice when compared with controls (Figure 4E). This ratio was significantly reduced in $S m o x^{-/}$mice that were uninfected/untreated, infected with C. rodentium, or treated with DSS compared with WT animals (Figure 4D). These results indicate that polyamine synthesis and metabolism is enhanced during colitis and that deletion of Smox 

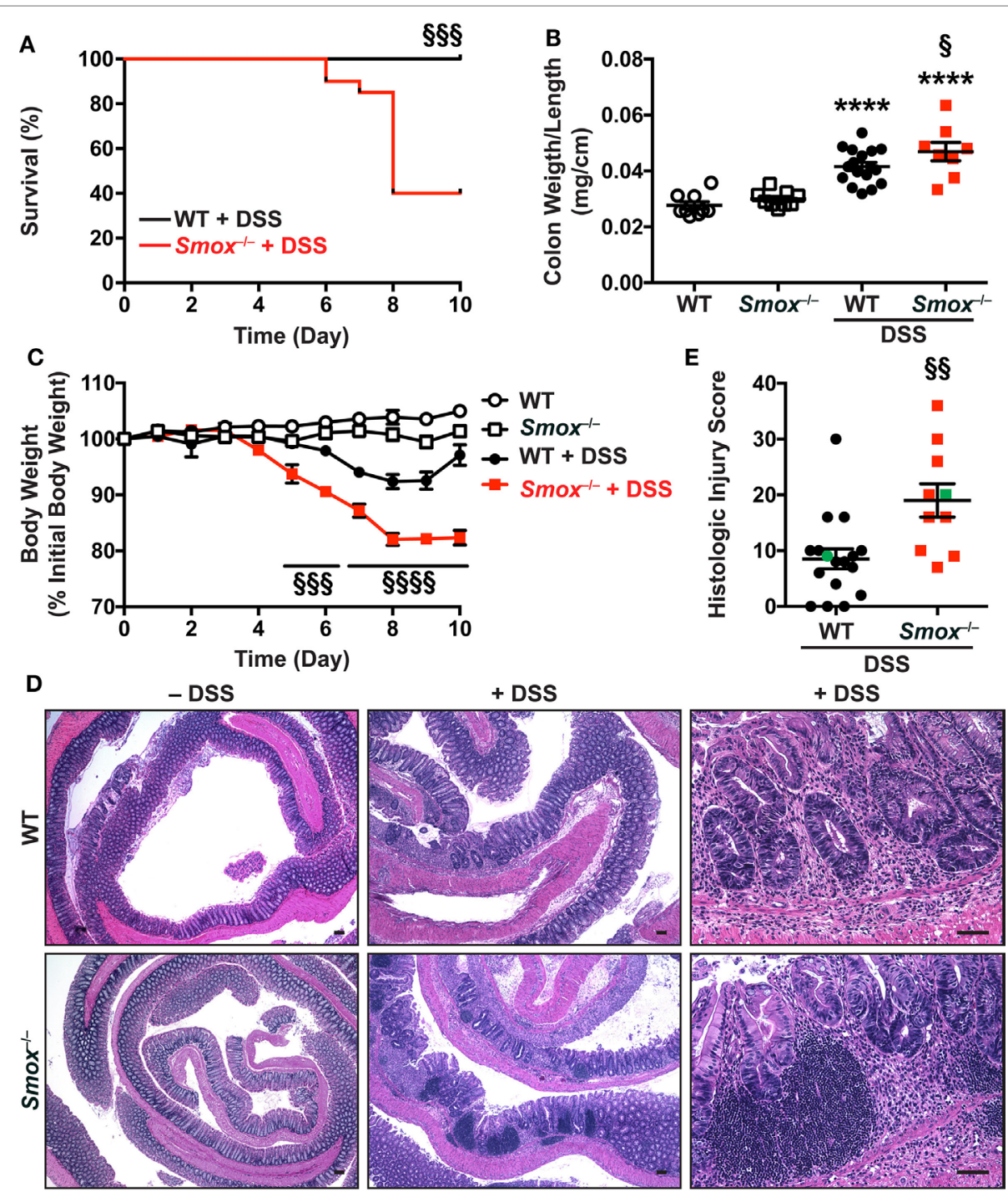

FIGURE 2 | Dextran sulfate sodium (DSS) colitis in C57BL/6 mice and Smox ${ }^{-/-}$mice. Animals were treated with $2.5 \%$ DSS for 5 days and then kept for 5 more days. (A) Survival was monitored daily; the Kaplan-Meier plot was performed from two separate experiments $\left[n=18\right.$ wild-type $\left(\right.$ WT) and $n=20$ Smox ${ }^{-/-}$mice]. (B) Colons were harvested, measured, washed, and weighed. (C) Body weights of all the animals in panel (A) were measured daily and are presented as percentage of initial body weight. (D,E) Colons were Swiss-rolled and stained with hematoxylin and eosin (D) and scored for histologic injury (E). The histologic injury score of untreated WT and Smox ${ }^{-/-}$mice was 0 for both. In panel (E), the green points represent the animals depicted in panel (D). Scale bar, 50 um. In all panels, ${ }^{\star \star \star \star} P<0.0001$ compared with uninfected animals; ${ }^{\S} P<0.05$, ${ }^{\S \S} P<0.01,{ }^{\S \S \S} P<0.001$, and ${ }^{\S \S \S} P<0.0001$ versus DSS-treated WT mice.

mainly leads to a diminution of spermidine concentration in the colon, which can likely be attributed to reduced back conversion of spermine to spermidine.

To gain more insight into the role of polyamines in the establishment of C. rodentium and DSS colitis, we correlated the histopathology score with the concentration of each polyamine for each WT mouse. In the $C$. rodentium infection model, we found a positive and significant correlation between putrescine, spermidine, and spermine concentration with the injury score (Figure 5A). In contrast, we found that spermidine level in the colon was inversely correlated with histologic injury score in the DSS model (Figure 5B); thus, more spermidine is associated with decreased DSS colitis (Figure 5B). There were no significant associations between the concentration of putrescine or spermine with DSS-mediated injury (Figure 5B). These data suggest that increased polyamine synthesis is deleterious for C. rodentiuminduced colitis, whereas spermidine protects mice during DSSinduced colitis.

\section{DISCUSSION}

In this study, we present compelling evidence that polyamines, and more particularly spermidine, can be considered as either beneficial or deleterious in colitis, depending on the etiology. In fact, in $C$. rodentium colitis, all three polyamines are associated with tissue damage and increased mucosal inflammation. 

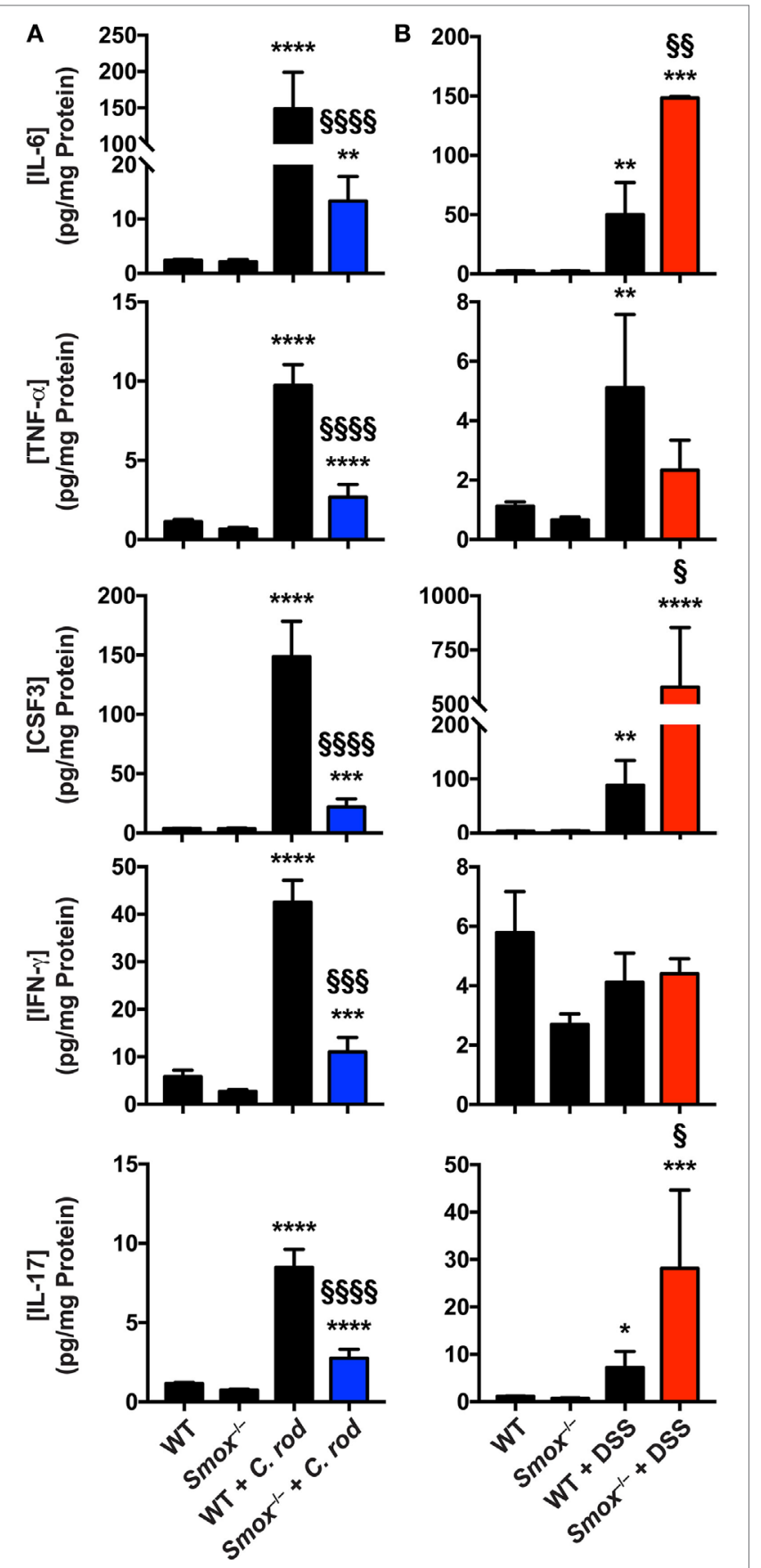

FIGURE 3 | Cytokines and chemokines in colonic tissues. Protein lysates from the colons of naïve wild-type (WT) and Smox ${ }^{-/-}$mice as well as Citrobacter rodentium-infected mice (A) and dextran sulfate sodium (DSS)-treated animals (B) were used to determine the concentration of cytokines and chemokines by Luminex assay. ${ }^{\star} P<0.05$, ${ }^{*} P<0.01$, ${ }^{\star \star \star} P<0.001$, and ${ }^{\star \star \star \star} P<0.0001$ compared with uninfected animals; $\S P<0.05$, $\$ \S P<0.01$, $\$ \S \S P<0.001$, and $\$ \S \S P<0.0001$ compared with WT mice infected with $C$. rodentium infection or DSS colitis.

By contrast, the generation of spermidine protects mice from DSSinduced colitis. In accordance with this result, we also found that knockout of Smox results in spermidine depletion and spermine accumulation in the colonic mucosa, which is associated with amelioration of C. rodentium colitis and worsening of DSSinduced colonic inflammation. Our data also indicate that these two models of colitis are driven by distinct pathological processes that can be potentially manipulated by the regulation of spermidine and spermine concentration.

Our laboratory has previously reported that SMOX is induced during $H$. pylori infection in macrophages $(14,31)$ and in gastric epithelial cells $(9,17)$. This leads to $\mathrm{H}_{2} \mathrm{O}_{2}$-mediated DNA damage $(9,17)$ and support of chronic inflammation, potentially due to spermine depletion (31). C. rodentium infection leads to the induction of ODC in the colon of mice $(7,27)$, which has been further underlined in this study by the increase of putrescine concentration in the colonic tissues of infected mice. However, we now observed that Smox is not induced in the colon of mice infected with $C$. rodentium. The discrepancy between $H$. pylori and $C$. rodentium in their ability to induce SMOX could be explained by the fact that SMOX induction in gastric tissue is dependent on the $H$. pylori virulence factor CagA (9), which is directly injected into host epithelial cells by a type IV secretion system. We can therefore postulate that $C$. rodentium does not possess the arsenal to stimulate Smox mRNA expression despite its numerous virulence factors including type III secretion system-dependent effectors (32). Contrasting to the C. rodentium model, we observed that Smox mRNA is upregulated in the colon of mice with DSS colitis. This is consistent with the increase of SMOX mRNA levels and SMOX protein expression in inflammatory cells of patients with ulcerative colitis (UC) (22). Similarly, Weiss et al. showed that spermidine was enhanced, and spermine was decreased in colonic epithelial cells of DSS-treated mice compared with untreated animals and in UC patients compared with healthy individuals (33), which is consistent with increased SMOX activity. Therefore, it would be of interest to determine which signaling pathways are involved in Smox mRNA expression during DSS colitis in future studies.

Spermine oxidase converts spermine into spermidine. Despite the fact that this enzyme was not upregulated in the colon of mice infected with $C$. rodentium, we found that Smox gene deletion resulted in a decreased concentration of spermidine and an accumulation of spermine in the colon. Similar data were obtained in the DSS model. Importantly, this effect of Smox deletion was also observed in uninfected/untreated animals. Altogether, these results support that the constitutive expression of SMOX, which can be augmented under specific conditions, is critical for the maintenance of spermine to spermidine flux in the colon and thus plays a role in the pathophysiology of colitis.

The next essential finding highlighted by our study is the dual role of polyamines in our two models of colitis. First, in WT mice infected with C. rodentium, increasing concentration of putrescine, spermidine, and spermine was significantly correlated with enhanced colonic injury score. Conversely, there was an inverse correlation between the damage to the colon and spermidine concentration in WT mice treated with DSS. These data therefore imply that polyamines are deleterious during C. rodentium infection and that spermidine protects animals against DSS-induced colitis. Consistently, we also observed that Smox deletion, which results in a reduction of spermidine concentration in the colon, is protective in C. rodentium colitis and exacerbates DSS-mediated 

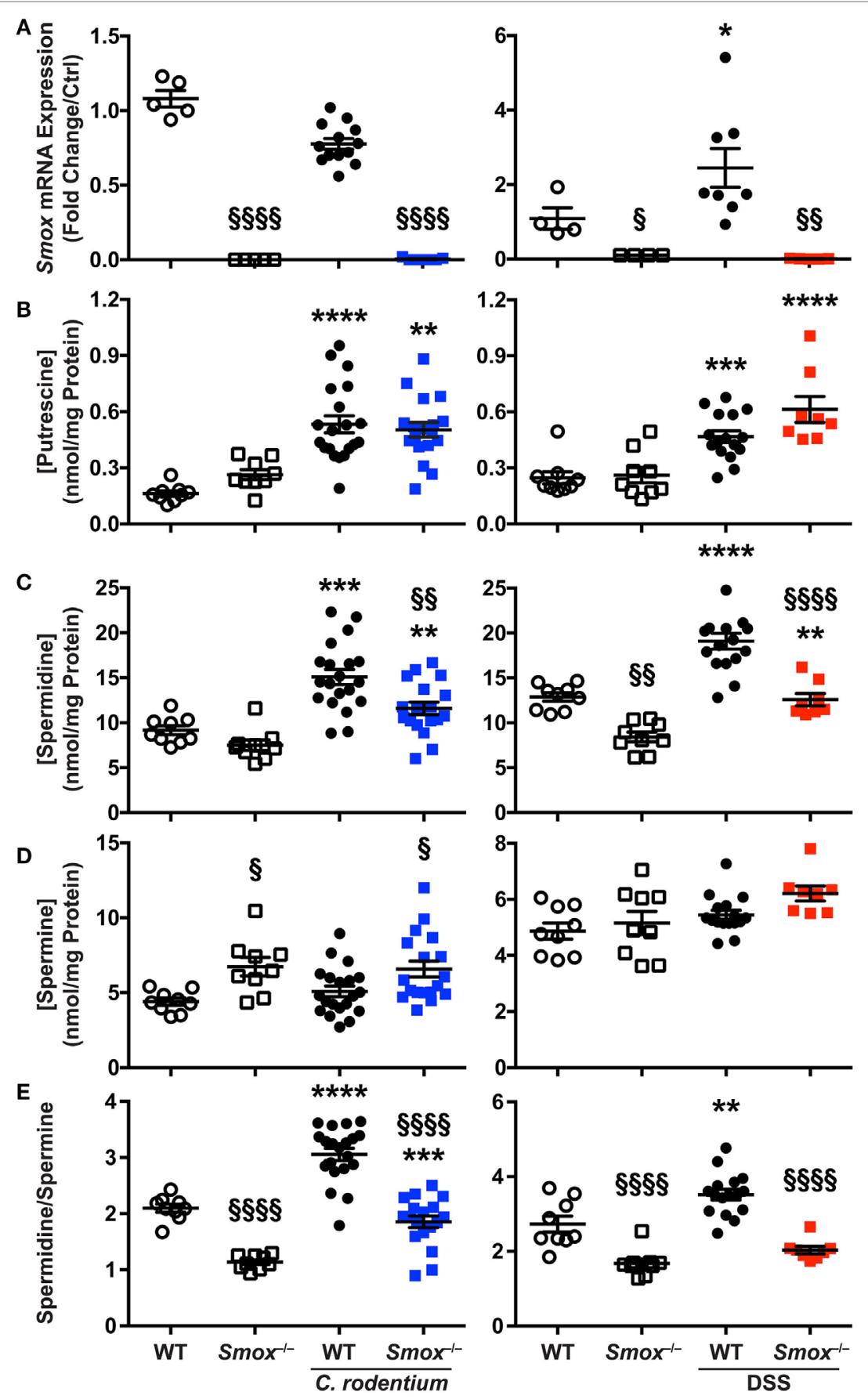

FIGURE 4 | Smox expression and polyamine profiles during murine colitis. (A) Smox mRNA expression in the colon. (B-D) The concentration of putrescine (B), spermidine (C), and spermine (D) was determined by LC/MS in the colonic tissues. (E) The spermidine/spermine ratio was calculated using the values depicted in

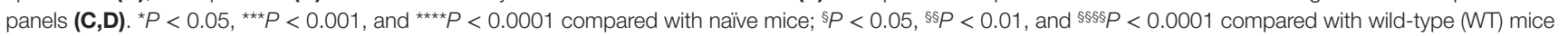
infected with Citrobacter rodentium infection or treated with dextran sulfate sodium (DSS).

injury. Although we cannot completely exclude a potential role for putrescine and spermine in the development of $C$. rodentiuminduced colitis, our data support the contention that spermidine displays opposite effects in mice with intestinal infection with an attaching and effacing pathogen or with epithelial injury-driven colitis.
The question raised by our finding is how can the same polyamine have differing effects in the colon depending on the model? Supporting the concept that spermidine protects from DSS colitis, it has been reported that this polyamine exhibits anti-inflammatory properties by dampening MAPKs, PI3K, STAT-1, and NF- $\mathrm{BB}$ signaling pathways in myeloid cells (34-36) 

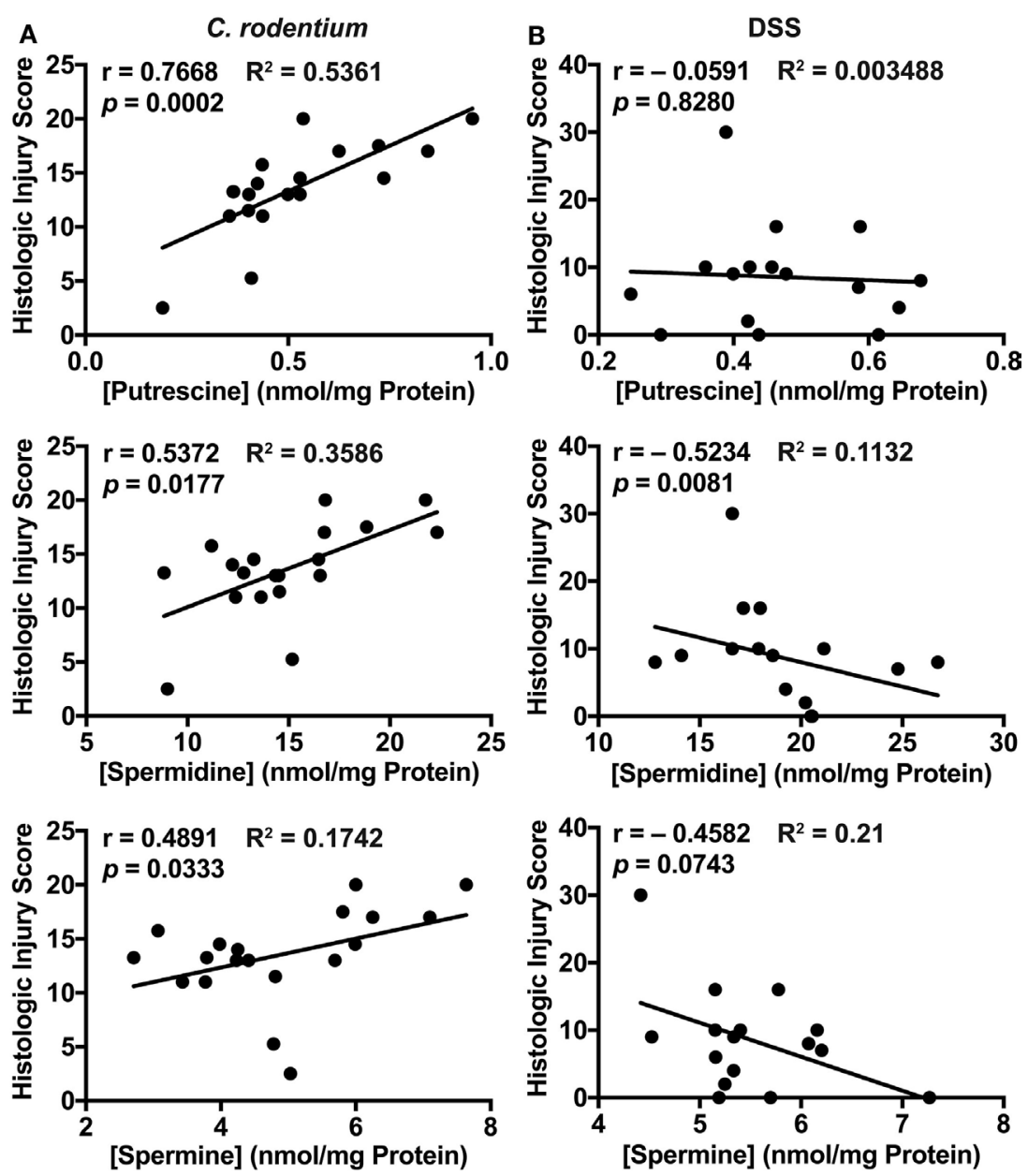

FIGURE 5 | Correlation plots comparing histologic injury scores of wild-type mice (from Figures 1D and 2E) and polyamine concentrations (from Figure 4) during Citrobacter rodentium (A) or dextran sulfate sodium (B) colitis. Statistical analysis was performed using the Pearson correlation test.

and thus gene transcription. Intriguingly, IFN- $\gamma$-induced synthesis of IL-6 is reduced in spermidine-treated THP-1 cells (35). This occurs through the spermidine-dependent stimulation of protein tyrosine phosphatase non-receptor type $2 \mathrm{a}$ that inhibits IFN- $\gamma$ signaling (35). Consistent with these prior findings, it should be noted that IL- 6 was one of the cytokines that we found to be further increased in Smox ${ }^{-/-}$mice during DSS colitis. Moreover, it has been described that daily supplementation of DSS-treated mice with spermidine improves clinical and histological parameters compared with sham-treated animals (35). In the same way, Yang et al. established that spermidine alleviates inflammation by favoring the polarization of macrophages toward an $\mathrm{M} 2$ response in a murine model of multiple sclerosis (37). The effect of spermidine on $C$. rodentium virulence gene expression is unknown and has not been analyzed in the present study. Intriguingly, it has been reported that spermidine is required for the virulence of Salmonella enterica Typhimurium in mice (38). Therefore, it could be now of interest whether decreased spermidine concentration in the colons of infected Smox $x^{-/}$mice could lead to a reduction of $C$. rodentium virulence, and consequently to less damage without affecting colonization levels. However, the potential role of putrescine and spermine on the pathophysiology of $C$. rodentium infection should be also considered, since the concentration of all three polyamines was significantly correlated with the damage to the colon.

In conclusion, our work reveals that the local homeostasis of spermidine and spermine through SMOX is a critical regulator of colonic inflammation. In the DSS model, spermidine is protective and consequently Smox deletion worsens colitis. Since SMOX is induced in the colon of UC patients $(22,33)$, we suggest that its induction could be beneficial by increasing spermidine concentration rather than deleterious by generating $\mathrm{H}_{2} \mathrm{O}_{2}$. Since DSS colitis exhibits some features similar to human UC, such patients might benefit from strategies to increase spermidine concentration in the colon, rather than inhibiting SMOX. Given that spermidine supplementation is safe, may also benefit the cardiovascular system (39) and exhibits neuroprotective and anti-cancerous properties (40), the use of this polyamine as a dietary supplement should be taken into consideration for patients with UC. However, our work 
also underlines that spermidine supplementation most likely should be avoided in the case of gastrointestinal infections, especially those involving attaching and effacing pathogens. Since different foods contain varying amount of spermidine (41), specific dietary recommendations related to polyamine intake may help in preventing colonic inflammation and/or severe intestinal infection.

\section{ETHICS STATEMENT}

Experimental procedures with animals were conducted under protocol M/08/124 approved by the IACUC of Vanderbilt University and the Research and Development Committee of the Veterans Affairs Tennessee Valley Healthcare System. Procedures were performed in accordance with institutional policies, AAALAC guidelines, the AVMA Guidelines on Euthanasia $\left(\mathrm{CO}_{2}\right.$ asphyxiation followed by cervical dislocation), NIH regulations (Guide for the Care and Use of Laboratory Animals), and the United States Animal Welfare Act (1966).

\section{AUTHOR CONTRIBUTIONS}

NA-G, AG, and KW designed research. NA-G, KS, JS, TV, PL, CS, MA, MMA, DB, MW, and MP performed experiments. JC,

\section{REFERENCES}

1. Pegg AE. Mammalian polyamine metabolism and function. IUBMB Life (2009) 61:880-94. doi:10.1002/iub.230

2. Igarashi K, Kashiwagi K. Modulation of protein synthesis by polyamines. IUBMB Life (2015) 67:160-9. doi:10.1002/iub.1363

3. Miller-Fleming L, Olin-Sandoval V, Campbell K, Ralser M. Remaining mysteries of molecular biology: the role of polyamines in the cell. J Mol Biol (2015) 427:3389-406. doi:10.1016/j.jmb.2015.06.020

4. Ramani D, De Bandt JP, Cynober L. Aliphatic polyamines in physiology and diseases. Clin Nutr (2014) 33:14-22. doi:10.1016/j.clnu.2013.09.019

5. Pegg AE. Functions of polyamines in mammals. JBiol Chem (2016) 291:14904-12. doi:10.1074/jbc.R116.731661

6. Pegg AE. Toxicity of polyamines and their metabolic products. Chem Res Toxicol (2013) 26:1782-800. doi:10.1021/tx400316s

7. Gobert AP, Cheng Y, Akhtar M, Mersey BD, Blumberg DR, Cross RK, et al. Protective role of arginase in a mouse model of colitis. J Immunol (2004) 173:2109-17. doi:10.4049/jimmunol.173.3.2109

8. Bussiere FI, Chaturvedi R, Cheng Y, Gobert AP, Asim M, Blumberg DR, et al. Spermine causes loss of innate immune response to Helicobacter pylori by inhibition of inducible nitric-oxide synthase translation. J Biol Chem (2005) 280:2409-12. doi:10.1074/jbc.C400498200

9. Chaturvedi R, Asim M, Romero-Gallo J, Barry DP, Hoge S, de Sablet T, et al. Spermine oxidase mediates the gastric cancer risk associated with Helicobacter pylori CagA. Gastroenterology (2011) 141:1696-708:e1-2. doi:10.1053/j.gastro. 2011.07.045

10. Gobert AP, Wilson KT. Polyamine- and NADPH-dependent generation of ROS during Helicobacter pylori infection: a blessing in disguise. Free Radic Biol Med (2017) 105:16-27. doi:10.1016/j.freeradbiomed.2016.09.024

11. Seiler N. Oxidation of polyamines and brain injury. Neurochem Res (2000) 25:471-90. doi:10.1023/A:1007508008731

12. Linsalata M, Russo F, Notarnicola M, Berloco P, Di Leo A. Polyamine profile in human gastric mucosa infected by Helicobacter pylori. Ital J Gastroenterol Hepatol (1998) 30:484-9.

13. Tunici P, Sessa A, Rabellotti E, Grant G, Bardocz S, Perin A. Polyamine oxidase and tissue transglutaminase activation in rat small intestine by polyamines. Biochim Biophys Acta (1999) 1428:219-24. doi:10.1016/S0304-4165 (99)00091-4
CDS, and RC developed and provided Smox ${ }^{-/-}$mice. AG and KW wrote the manuscript.

\section{FUNDING}

This work was funded by NIH grants R01AT004821, R01CA190612, P01CA116087, P01CA028842 (KW), RO1CA204345 (RC); Maryland Cigarette Restitution Fund (RC); Veterans Affairs Merit Review grant I01BX001453 (KW); the Thomas F Frist Sr. Endowment (KW); and the Vanderbilt Center for Mucosal Inflammation and Cancer (KW). LC was supported by Veterans Affairs Career Development Award 1IK2BX002126. This work was supported by NIH awards AT006896 by NCCIH (CS and PL) was supported by a postdoctoral fellowship award from the American Heart Association (16POST27250138). Mass spectrometric analyses were in part performed through Vanderbilt University Medical Center's Digestive Disease Research Center supported by NIH grant P30DK058404 Core Scholarship.

\section{SUPPLEMENTARY MATERIAL}

The Supplementary Material for this article can be found online at https://www.frontiersin.org/articles/10.3389/fimmu.2018.01242/ full\#supplementary-material.

14. Chaturvedi R, Cheng Y, Asim M, Bussiere FI, Xu H, Gobert AP, et al. Induction of polyamine oxidase 1 by Helicobacter pylori causes macrophage apoptosis by hydrogen peroxide release and mitochondrial membrane depolarization. J Biol Chem (2004) 279:40161-73. doi:10.1074/jbc.M401370200

15. Wang Y, Devereux W, Woster PM, Stewart TM, Hacker A, Casero RA Jr. Cloning and characterization of a human polyamine oxidase that is inducible by polyamine analogue exposure. Cancer Res (2001) 61:5370-3.

16. Goodwin AC, Destefano Shields CE, Wu S, Huso DL, Wu X, Murray-Stewart TR, et al. Polyamine catabolism contributes to enterotoxigenic Bacteroides fragilisinduced colon tumorigenesis. Proc Natl Acad Sci U S A (2011) 108:15354-9. doi:10.1073/pnas.1010203108

17. Xu H, Chaturvedi R, Cheng Y, Bussiere FI, Asim M, Yao MD, et al. Spermine oxidation induced by Helicobacter pylori results in apoptosis and DNA damage: implications for gastric carcinogenesis. Cancer Res (2004) 64:8521-5. doi:10.1158/0008-5472.CAN-04-3511

18. Chaturvedi R, Asim M, Piazuelo MB, Yan F, Barry DP, Sierra JC, et al. Activation of EGFR and ERBB2 by Helicobacter pylori results in survival of gastric epithelial cells with DNA damage. Gastroenterology (2014) 146: 1739-51. doi:10.1053/j.gastro.2014.02.005

19. Chaturvedi R, de Sablet T, Asim M, Piazuelo MB, Barry DP, Verriere TG, et al. Increased Helicobacter pylori-associated gastric cancer risk in the Andean region of Colombia is mediated by spermine oxidase. Oncogene (2015) 34:3429-40. doi:10.1038/onc.2014.273

20. Shivashankar R, Tremaine WJ, Harmsen WS, Loftus EV Jr. Incidence and prevalence of Crohn's disease and ulcerative colitis in Olmsted County, Minnesota from 1970 through 2010. Clin Gastroenterol Hepatol (2017) 15:857-63. doi:10.1016/j.cgh.2016.10.039

21. Singh P, Ananthakrishnan A, Ahuja V. Pivot to Asia: inflammatory bowel disease burden. Intest Res (2017) 15:138-41. doi:10.5217/ir.2017.15.1.138

22. Hong SK, Chaturvedi R, Piazuelo MB, Coburn LA, Williams CS, Delgado AG, et al. Increased expression and cellular localization of spermine oxidase in ulcerative colitis and relationship to disease activity. Inflamm Bowel Dis (2010) 16:1557-66. doi:10.1002/ibd.21224

23. Zahedi K, Barone S, Destefano-Shields C, Brooks M, Murray-Stewart T, Dunworth $\mathrm{M}$, et al. Activation of endoplasmic reticulum stress response by enhanced polyamine catabolism is important in the mediation of cisplatininduced acute kidney injury. PLoS One (2017) 12:e0184570. doi:10.1371/journal. pone. 0184570 
24. Barthold SW, Coleman GL, Bhatt PN, Osbaldiston GW, Jonas AM. The etiology of transmissible murine colonic hyperplasia. Lab Anim Sci (1976) 26: 889-94.

25. Singh K, Chaturvedi R, Barry DP, Coburn LA, Asim M, Lewis ND, et al. The apolipoprotein E-mimetic peptide COG112 inhibits NF-kappaB signaling, proinflammatory cytokine expression, and disease activity in murine models of colitis. J Biol Chem (2011) 286:3839-50. doi:10.1074/jbc.M110.176719

26. Singh K, Al-Greene NT, Verriere TG, Coburn LA, Asim M, Barry DP, et al. The L-arginine transporter solute carrier family 7 member 2 mediates the immunopathogenesis of attaching and effacing bacteria. PLoS Pathog (2016) 12:e1005984. doi:10.1371/journal.ppat.1005984

27. Hardbower DM, Asim M, Luis PB, Singh K, Barry DP, Yang C, et al. Ornithine decarboxylase regulates M1 macrophage activation and mucosal inflammation via histone modifications. Proc Natl Acad Sci U S A (2017) 114:E751-60. doi:10.1073/pnas.1614958114

28. Coburn LA, Gong X, Singh K, Asim M, Scull BP, Allaman MM, et al. L-arginine supplementation improves responses to injury and inflammation in dextran sulfate sodium colitis. PLoS One (2012) 7:e33546. doi:10.1371/ journal.pone.0033546

29. Singh K, Coburn LA, Barry DP, Asim M, Scull BP, Allaman MM, et al. Deletion of cationic amino acid transporter 2 exacerbates dextran sulfate sodium colitis and leads to an IL-17-predominant T cell response. Am J Physiol Gastrointestin Liver Physiol (2013) 305:G225-40. doi:10.1152/ajpgi.00091.2013

30. Hardbower DM, Singh K, Asim M, Verriere TG, Olivares-Villagomez D, Barry DP, et al. EGFR regulates macrophage activation and function in bacterial infection. J Clin Invest (2016) 126:3296-312. doi:10.1172/JCI83585

31. Chaturvedi R, Asim M, Barry DP, Frye JW, Casero RA Jr, Wilson KT. Spermine oxidase is a regulator of macrophage host response to Helicobacter pylori: enhancement of antimicrobial nitric oxide generation by depletion of spermine. Amino Acids (2014) 46:531-42. doi:10.1007/s00726-013-1531-z

32. Deng W, Puente JL, Gruenheid S, Li Y, Vallance BA, Vazquez A, et al. Dissecting virulence: systematic and functional analyses of a pathogenicity island. Proc Natl Acad Sci U S A (2004) 101:3597-602. doi:10.1073/pnas.0400326101

33. Weiss TS, Herfarth H, Obermeier F, Ouart J, Vogl D, Scholmerich J, et al. Intracellular polyamine levels of intestinal epithelial cells in inflammatory bowel disease. Inflamm Bowel Dis (2004) 10:529-35. doi:10.1097/00054725200409000-00006
34. Choi YH, Park HY. Anti-inflammatory effects of spermidine in lipopolysaccharide-stimulated BV2 microglial cells. JBiomed Sci (2012) 19:31. doi:10.1186/1423-0127-19-31

35. Moron B, Spalinger M, Kasper S, Atrott K, Frey-Wagner I, Fried M, et al. Activation of protein tyrosine phosphatase non-receptor type 2 by spermidine exerts anti-inflammatory effects in human THP-1 monocytes and in a mouse model of acute colitis. PLoS One (2013) 8:e73703. doi:10.1371/journal. pone.0073703

36. Jeong JW, Cha HJ, Han MH, Hwang SJ, Lee DS, Yoo JS, et al. Spermidine protects against oxidative stress in inflammation models using macrophages and zebrafish. Biomol Ther (Seoul) (2018) 26:146-56. doi:10.4062/biomolther.2016.272

37. Yang Q, Zheng C, Cao J, Cao G, Shou P, Lin L, et al. Spermidine alleviates experimental autoimmune encephalomyelitis through inducing inhibitory macrophages. Cell Death Differ (2016) 23:1850-61. doi:10.1038/cdd.2016.71

38. Espinel IC, Guerra PR, Jelsbak L. Multiple roles of putrescine and spermidine in stress resistance and virulence of Salmonella enterica serovar Typhimurium. Microb Pathog (2016) 95:117-23. doi:10.1016/j.micpath.2016.03.008

39. Eisenberg T, Abdellatif M, Schroeder S, Primessnig U, Stekovic S, Pendl T, et al. Cardioprotection and lifespan extension by the natural polyamine spermidine. Nat Med (2016) 22:1428-38. doi:10.1038/nm.4222

40. Madeo F, Eisenberg T, Pietrocola F, Kroemer G. Spermidine in health and disease. Science (2018) 359:eaan2788. doi:10.1126/science.aan2788

41. Atiya Ali M, Poortvliet E, Stromberg R, Yngve A. Polyamines in foods: development of a food database. Food Nutr Res (2011) 55:1. doi:10.3402/fnr.v55i0.5572

Conflict of Interest Statement: The authors declare that the research was conducted in the absence of any commercial or financial relationships that could be construed as a potential conflict of interest.

Copyright (C) 2018 Gobert, Al-Greene, Singh, Coburn, Sierra, Verriere, Luis, Schneider, Asim, Allaman, Barry, Cleveland, Destefano Shields, Casero, Washington, Piazuelo and Wilson. This is an open-access article distributed under the terms of the Creative Commons Attribution License (CC BY). The use, distribution or reproduction in other forums is permitted, provided the original author(s) and the copyright owner are credited and that the original publication in this journal is cited, in accordance with accepted academic practice. No use, distribution or reproduction is permitted which does not comply with these terms. 